\title{
Roux-en-Y-Bariatric Surgery Reduces Markers of Metabolic Syndrome in Morbidly Obese Patients
}

\author{
G. Rega-Kaun $^{1,2} \cdot$ C. Kaun ${ }^{1} \cdot$ G. Jaegersberger ${ }^{1,3} \cdot$ M. Prager ${ }^{4,5} \cdot$ M. Hackl $^{6} \cdot$ S. Demyanets ${ }^{1,7} \cdot$ J. Wojta $^{1,3,8}$ (D) \\ P. J. Hohensinner ${ }^{1,3}$
}

Published online: 15 November 2019

(C) The Author(s) 2019

\begin{abstract}
Background Obesity is closely linked to increased markers of metabolic syndrome and development of diabetes. Roux-en-Y bariatric surgery reduces hyperinsulinemia and improves insulin sensitivity and hence benefits morbidly obese patients.

Aim To determine changes in markers of metabolic syndrome, pancreatic function, and hepatic insulin sensitivity in patients before and 1 year after undergoing Roux-en-Y gastric bypass surgery.

Methods We enrolled 43 consecutive patients in a single center. Markers for metabolic syndrome included proinsulin, insulin, Cpeptide, liver enzymes, and serum levels of selected microRNAs hsa-miR-122, hsa-miR-130, hsa-miR-132, and hsa-miR-375. Results After surgery, all patients showed a significant $37 \%$ drop of body mass index $(p<0.001)$. Furthermore, proinsulin $(59 \%$ reduction, $p<0.001$ ), insulin (76\% reduction, $p<0.001$ ), and C-peptide (56\% reduction, $p<0.001)$ were all reduced 1 year after surgery. Using the hepatic insulin clearance score, we determined a significant increase in hepatic insulin clearance after surgery (76\% increase, $p<0.001$ ). Especially diabetic patients showed a marked 2.1-fold increase after surgery. Hepatic enzymes ALT (35\% reduction, $p=0.002$ ) and $\gamma \mathrm{GT}$ (48\% reduction, $p<0.001)$ were significantly reduced in all patients with similar improvement in diabetic and non-diabetic patients. miRNAs hsa-miR-122, hsa-miR-130, and hsa-miR-132 were all significantly reduced whereas hsa-miR-375 was increased after gastric bypass surgery $(p<0.001$ for all miRNAs).

Conclusion Both liver and pancreatic stress parameters were reduced significantly 1 year after Roux-en-Y gastric bypass surgery suggesting an overall amelioration of the metabolic syndrome in all patients regardless of previous health status.
\end{abstract}

Keywords Bariatric surgery · Metabolic syndrome $\cdot$ miRNA $\cdot$ Insulin sensitivity $\cdot$ Hepatic insulin clearance

J. Wojta

johann.wojta@meduniwien.ac.at

G. Rega-Kaun

gersina.rega-kaun@meduniwien.ac.at

C. Kaun

christoph.kaun@meduniwien.ac.at

G. Jaegersberger

gerlinde.jaegersberger@meduniwien.ac.at

M. Prager

manfred.prager@wienkav.at

M. Hackl

matthias.hack1@tamirna.com

S. Demyanets

svitlana.demyanets@meduniwien.ac.at
P. J. Hohensinner

philipp.hohensinner@meduniwien.ac.at

1 Department of Internal Medicine II, Division of Cardiology, Medical University of Vienna, Waehringer Guertel 18-20, 1090 Vienna, Austria

2 5th Medical Department for Endocrinology and Rheumatology, Wilhelminenhospital, Vienna, Austria

3 Ludwig Boltzmann Institute for Cardiovascular Research, Vienna, Austria

4 Department of Surgery, Hospital Hietzing, Vienna, Austria

5 Department of Surgery, Hospital Oberwart, Oberwart, Austria

6 TAmiRNA GmbH, Vienna, Austria

7 Department of Laboratory Medicine, Medical University of Vienna, Vienna, Austria

8 Core Facilities, Medical University of Vienna, Vienna, Austria 


\section{Introduction}

Increased risk of developing type 2 diabetes is linked to an accumulation of several metabolic abnormalities [1]. Among them, insulin resistance and hyperinsulinemia are the core and fundamental sign of metabolic syndrome [2]. Besides insulin resistance, also markers of systemic inflammation, hypercoagulability, and increased liver enzymes are considered part of the metabolic syndrome [1]. Obesity is closely linked to markers of the metabolic syndrome and the development of type 2 diabetes [3]. A hallmark of insulin resistance in obesity is hyperinsulinemia and the interplay between increased insulin secretion and insulin resistance either due to a compensatory mechanism or secondary resistance development $[4,5]$. The clearance of insulin is mainly by the liver and therefore hepatic insulin clearance contributes to the circulating levels of insulin [6]. Bariatric surgery has become an effective treatment for morbid obesity including an improvement in glucose metabolism, insulin resistance, and reduction of proinsulin levels [7-10]. In addition to changes in the metabolic syndrome, bariatric surgery is also associated with a reduced inflammatory profile and amelioration of coagulation [11, 12].

When analyzing the pancreas in obesity, pancreatic fat accumulation is thought to accompany beta cell failure at least in animal models [13]. Recent data described a normalization of pancreatic volume and steatosis in morbidly obese patients following Roux-en-Y gastric bypass (RYGB) surgery [14]. Under normal physiologic conditions, insulin biosynthesis is a multi-step process beginning with a pre-prohormone which is converted in the rough endoplasmatic reticulum to proinsulin within beta cells [15]. Glucose is both an inducer for proinsulin biosynthesis and for regulation of insulin secretion [16]. Due to the increased metabolic load and insulin resistance caused by obesity, resulting pressure on the beta cell for increased insulin production can lead to the development of a variety of beta cell stresses [17]. This can lead to an increased release of unprocessed proinsulin in type 2 diabetes [18]. Increased proinsulin secretion can therefore be a biomarker for beta cell dysfunction [19]. Besides pancreatic dysfunction, morbid obesity is also associated with liver dysfunction which can be ameliorated by RYGB surgery [20]. This liver dysfunction could further link back to increased insulin levels as hepatic clearance of insulin is reduced during obesity [1].

Besides classical laboratory parameters and protein data, also circulating micro-ribonucleic acids (miRNAs) are potential sensors of organ function. miRNAs are small, non-coding RNAs that bind to the $3^{\prime}$ untranslated region of mRNAs of specific genes to prevent their translation [21]. They act as post-transcriptional regulators of gene expression [22]. As such, miRNAs regulate a multitude of processes, especially as miRNAs have numerous mRNA targets. Based on active or passive release from cells into the circulation and the fact that some miRNAs are closely connected to the function of specific organs, they are considered potentially important minimal-invasive biomarkers.

Within our study, we analyzed changes associated with the metabolic syndrome 1 year after RYGB surgery. We determined markers for insulin sensitivity both from the pancreas and the liver. We further evaluated the expression profile of the selected miRNAs hsa-miR-122, hsa-miR-130, hsa-miR132 , and hsa-miR-375 for the study of hepatic and pancreatic changes after surgery.

\section{Methods}

\section{Patient Recruitment and Sampling}

This was a single-center, prospective observational study with the same surgeon responsible for all surgical interventions. Study participants were enrolled after selection for RYGB surgery following the respective guidelines [23]. All procedures were performed by the same surgical team (M.P.) using a laparoscopic approach. Roux-en-Y gastric bypass consists of a longitudinal 30-40-ml gastric pouch which was anastomosed end-to-side with the jejunal limb using a circular anastomotic stapler with a diameter of $21 \mathrm{~mm}$ and a latero-lateral jejuno-jejunal anastomosis resulting in a biliopancreatic limb of approximately $100 \mathrm{~cm}$ and an alimentary limb of approximately $150 \mathrm{~cm}$ [24]. The bypass surgery was done in a standardized technique; there were no individual adjustments to any kind of patient particularity. Patients were selected for the study on a consecutive basis if participating in the 12 months follow-up. A total of 26 additional patients did not participate in the 1 year follow-up but were present for a 6 months followup. All patients were Caucasian in accordance with the patient population in the recruiting hospital. Before surgery and at the 1 year follow-up visit, venous blood was drawn from the antecubital vein. After centrifugation (2800 rpm, $20 \mathrm{~min})$, plasma and serum samples were stored at $-80^{\circ} \mathrm{C}$ in multiple aliquots. Diabetic patients were defined as subjects already on anti-diabetic medication (metformin, sulfonylureas, and dipeptidyl peptidase 4 inhibitors were in use in the current patient cohort). Furthermore, remaining individuals underwent an oral glucose tolerance test to define their diabetic status identifying two patients with diabetes not known and not treated previously.

\section{Protein Determination}

Concentrations of high-sensitive C-reactive protein (hs-CRP) were measured using particle enhanced immunoturbidimetric assay on cobas ${ }^{\circledR} 8000$ modular analyzer (Cardiac C-Reactive Protein (Latex) High Sensitive, Roche Diagnostics, Germany). Concentrations of glucose, insulin, and C-peptide were measured at the Department of Laboratory Medicine, 
Medical University of Vienna. Insulin and C-peptide concentrations were determined using Electro-chemiluminescence immunoassay (ECLIA) on cobas ${ }^{\circledR}$ e analyzer (Roche Diagnostics). Concentrations of glucose were analyzed using the hexokinase method on cobas ${ }^{\circledR} 8000$ modular analyzer (Roche Diagnostics). Levels of proinsulin (Biotechne, USA), neuregulin (NRG)-4 (Abbexa, UK), total glucagon-like peptide-(GLP-) 1 (Mercodia, Sweden), and interleukin- (IL-) 10 (Thermo Fisher, USA) were determined by ELISA. Aspartate transaminase (AST), alanine transaminase (ALT), and $\gamma$-glutamyltransferase $(\gamma \mathrm{GT})$ were determined using routine assays on a cobas ${ }^{\circledR} 501$ instrument (Roche Diagnostics).

\section{miRNA Isolation and Determination}

miRNAs were isolated and determined as published previously [11]. Briefly, total RNAs including miRNAs were extracted from $200 \mu \mathrm{l}$ of EDTA plasma using an automated Maxwell system (Promega, USA) with the respective miRNA isolation kit (miRNA tissue lysis kit, Promega). Three synthetic spikein controls (Exiqon, Denmark) were added to the lysis buffer prior to isolation in order to monitor RNA extraction efficiency. Two microliter of total RNA was converted to cDNA using the universal cDNA synthesis kit II (Exiqon) at $42{ }^{\circ} \mathrm{C}$ for $60 \mathrm{~min}$, followed by inactivation at $95{ }^{\circ} \mathrm{C}$ for $5 \mathrm{~min}$. One microliter of cel-miR-39 was added to monitor the efficiency of reverse transcription. qPCR was set up in $10 \mu$ l reactions using ExiLENT SYBR® Green Mastermix (Exiqon) and LNA microRNA primer assays (Exiqon). PCR conditions were $95{ }^{\circ} \mathrm{C}$ for $10 \mathrm{~min}$ of activation followed by 45 cycles of denaturation $\left(95^{\circ} \mathrm{C}, 10 \mathrm{~s}\right)$ and annealing/elongation $\left(60^{\circ} \mathrm{C}\right.$, $60 \mathrm{~s}$ ), and melting curve analysis. PCR amplification and fluorescence detection were performed on a Roche LightCycler 480 II in 96-well plates. Hemolysis was checked using the ratio of miR-23a-3p vs. miR-451a, as described previously [25].

\section{Statistics}

Homeostasis model assessment 1 (HOMA1) index [26] and quantitative insulin sensitivity check index (QUICKI) [27] were calculated as described in the literature. To estimate the rate of hepatic clearance of insulin, we calculated the ratio of C-peptide and insulin as suggested [28]. Statistical calculations were performed using SPSS 25 (IBM, USA). Correlations are given using the Pearson correlation factor $R$ and were considered significant at $p \leq 0.05$. Changes were calculated by Wilcoxon rank test with significance assumed at $p \leq 0.05$ after testing parameters with a KolmogorovSmirnov test. Ratios were calculated by dividing the 1 year post surgery value by the pre-surgery value.

\section{Results}

To evaluate changes in metabolic syndrome-associated parameters in morbidly obese patients and their changes after RYGB surgery, we included a total of 43 patients undergoing bariatric surgery. Clinical parameters before and after surgery are given in Table 1 . The median age before surgery was 45.8 years with a majority of female participants (29 participants, 67\%). Of the 43 patients, 17 (40\%) reported with diabetes. Overall, laboratory parameters were mostly within the high range of normal values. After surgery, we observed a massive change for most of the analyzed parameters (Table 2). As expected, both weight and BMI dropped massively and significantly from a median BMI of 43.4 to 27.2 $(p<0.001)$. Cholesterol and LDL were reduced and HDL slightly increased 1 year after bariatric surgery. In parallel inflammation measured via hs-CRP dropped by a median of $88 \%$ and the anti-inflammatory marker IL-10 increased by $83 \% 1$ year after surgery. The adipokine neuregulin-4, which is associated with metabolism in animal models, remained unchanged by the procedure [29]. In addition, baseline levels of the postprandial GLP-1 were unaltered in the overall population. In contrast, proinsulin, insulin, and C-peptide levels dropped significantly by $59 \%, 76 \%$, and $56 \%$, respectively, after surgery. This resulted in a similarly robust $81 \%$ drop in HOMA1 and a slight increase in QUICKI by $26 \%$. When comparing the top $20 \%$ of patients regarding weight loss with the bottom $20 \%$, we found that patients with high weight loss showed a stronger reduction of insulin levels compared with patients with only low weight loss $(p=0.024)$. No effect was observed for proinsulin or C-peptide. Hepatic insulin clearance score (HICS) was increased by $76 \%$ suggesting improved hepatic insulin clearance. Interestingly, of all the tested parameters, only relative changes of proinsulin correlated with a reduction of BMI $(R=0.355, p=0.02)$ whereas changes of the remaining parameters were not associated with changes in BMI. The correlation of proinsulin with BMI was only

Table 1 General patient population

\begin{tabular}{lrr}
\hline Age at start & Median 45.8 & IQR 23.7 \\
Sex & Female 29 (67\%) & Male 14 (33\%) \\
Diabetic & $17(40 \%)$ & \\
Hypertension & $24(43 \%)$ & \\
Medication & Before surgery & After surgery \\
Statins & $11(26 \%)$ & $5(12 \%)$ \\
Antidiabetics & $13(30 \%)$ & $2(5 \%)$ \\
Insulin & $2(5 \%)$ & $1(2 \%)$ \\
ACE-inhibitors & $23(53 \%)$ & $13(30 \%)$ \\
Beta blockers & $11(26 \%)$ & $5(12 \%)$ \\
\hline
\end{tabular}

Characteristics of the study population regarding their starting baseline characteristics and medication including changes in medication 1 year after bariatric surgery 
Table 2 Patient characteristics before and 1 year after bariatric surgery

\begin{tabular}{|c|c|c|c|c|c|c|c|}
\hline & \multicolumn{2}{|c|}{ Before surgery } & \multicolumn{5}{|c|}{ After surgery } \\
\hline & Median & IQR & Median & IQR & Delta & IQR & $p$ value \\
\hline Weight $(\mathrm{kg})$ & 126 & 29 & 81 & 21 & 0.63 & 0.12 & $p<0.001$ \\
\hline BMI & 43.4 & 5.5 & 27.2 & 4.7 & 0.63 & 0 & $p<0.001$ \\
\hline Cholesterol (mg/dl) & 173 & 30 & 148 & 42 & 0.87 & 0.21 & $p<0.001$ \\
\hline HDL (mg/dl) & 44 & 12 & 54 & 18 & 1.22 & 0.35 & $p<0.001$ \\
\hline $\mathrm{LDL}(\mathrm{mg} / \mathrm{dl})$ & 95 & 32 & 75 & 32 & 0.81 & 0.34 & $p<0.001$ \\
\hline hs-CRP (mg/dl) & 0.47 & 0.65 & 0.06 & 0.2 & 0.12 & 0.26 & $p<0.001$ \\
\hline IL-10 (pg/ml) & 1.31 & 1.15 & 1.95 & 2.3 & 1.83 & 2.9 & $p<0.001$ \\
\hline NRG-4 (ng/ml) & 0.68 & 0.36 & 0.6 & 0.2 & 0.98 & 0.47 & $p=0.214$ \\
\hline GLP-1 (pmol/1) & 2.45 & 2.44 & 1.47 & 3.48 & 0.76 & 2.72 & $p=0.530$ \\
\hline Glucose (mg/dl) & 99 & 45 & 87 & 14 & 0.87 & 0.35 & $p<0.001$ \\
\hline Proinsulin (pM) & 10.78 & 10.96 & 4.86 & 4 & 0.41 & 0.36 & $p<0.001$ \\
\hline Insulin (U/ml) & 23.9 & 20.4 & 4.8 & 6.5 & 0.24 & 0.16 & $p<0.001$ \\
\hline C-Peptid (ng/ml) & 4.2 & 2 & 1.9 & 1.2 & 0.44 & 0.19 & $p<0.001$ \\
\hline HOMA1 & 5.33 & 7.63 & 1.12 & 1.5 & 0.19 & 0.2 & $p<0.001$ \\
\hline QUICKI & 0.3 & 0.05 & 0.38 & 0.1 & 1.26 & 0.21 & $p<0.001$ \\
\hline HICS & 0.19 & 0.11 & 0.38 & 0.1 & 1.76 & 0.9 & $p<0.001$ \\
\hline
\end{tabular}

Characteristics of study subjects before and 1 year after bariatric surgery. Values are given in median including the interquartile range (IQR). Delta values represent fold changes in values after surgery divided by values before surgery. $B M I$ body mass index, $H D L$ high-density lipoprotein, $L D L$ low-density lipoprotein, $h s$ - $C R P$ high-sensitive C-reactive protein, $I L-10$ interleukin 10, NRG-4 neuregulin 4, GLP-1 glucagon-like peptide-1, HOMAI homeostasis model assessment 1, QUICKI quantitative insulin sensitivity check index, HICS hepatic insulin clearance score. Statistical significance was determined using a Wilcoxon rank test; $p \leq 0.05$ was considered significant observed in diabetic patients $(R=0.525, p=0.031)$ and not in non-diabetic patients $(R=0.152, p=0.458)$. When analyzing correlations among insulin, proinsulin, and C-peptide, we found correlations of insulin with C-peptide $(R=0.786$, $p<0.001)$ and glucose $(R=0.485, p=0.001)$ but not with proinsulin $(R=0.102, p=0.513)$ before surgery whereas all parameters correlated with insulin after RYGB surgery (Cpeptide: $R=0.819, p<0.001$; glucose: $R=0.351, p=0.021$; proinsulin: $R=0.554, p<0.001)$. Of note, similar changes were observed in patients undergoing a 6-month follow-up (Table 3).

In order to better evaluate differences in diabetic and nondiabetic patients, we determined the respective changes according to the initial diabetic status of the respective patient (Table 4). Before surgery, we did not find differences between non-diabetic and diabetic patients regarding weight, BMI, cholesterol, HDL, LDL, hs-CRP, GLP-1, and IL-10. Interestingly, also NRG-4 was not different in our cohort. In contrast, we found significant differences in metabolic syndrome parameters as expected. Of note, proinsulin did not differ between non-diabetic and diabetic patients before surgery whereas hepatic insulin clearance was significantly higher in non-diabetic patients. After surgery, we found that diabetic patients still displayed higher insulin and C-peptide levels. However, hepatic insulin clearance was similar in nondiabetic and diabetic patients. When analyzing the relative change of values before and after bariatric surgery in dependence of diabetic status, we observed reduced loss of weight and hence BMI in diabetic patients. However, when comparing diabetic and non-diabetic patients, glucose levels were reduced more significantly in diabetic patients together with an increase in hepatic insulin clearance, which, however, did not reach statistical significance.

Table 3 Patient characteristics 6 months after bariatric surgery

\begin{tabular}{lccccccr}
\hline & \multicolumn{2}{l}{ Before surgery } & & \multicolumn{2}{l}{ After surgery } & \\
\cline { 2 - 3 } & Median & IQR & & Median & IQR & & $p$ value \\
\hline Weight (kg) & 129 & 20 & 91.12 & 19 & $<0.001$ \\
BMI & 44.9 & 3.4 & 30.9 & 5.5 & $<0.001$ \\
hs-CRP (mg/dl) & 0.69 & 0.89 & 0.21 & 0.36 & $<0.001$ \\
Glucose (mg/dl) & 93 & 15 & & 86 & 17 & 0.017 \\
Insulin (U/ml) & 18.6 & 11.1 & & 7.1 & 4 & $<0.001$ \\
C-Peptid (ng/ml) & 3.65 & 2.5 & & 2.1 & 0.72 & $<0.001$ \\
HOMA1 & 4.1 & 3.8 & & 1.5 & 1.2 & $<0.001$ \\
QUICKI & 0.31 & 0.04 & & 0.36 & 0.05 & $<0.001$ \\
HICS & 0.19 & 0.11 & & 0.29 & 0.12 & $<0.001$ \\
\hline
\end{tabular}

Characteristics of an additional 26 patients undergoing a 6-month followup but not a 1-year follow-up are given in Table 3. Statistical significance was determined using a Wilcoxon rank test; $p \leq 0.05$ was considered significant 


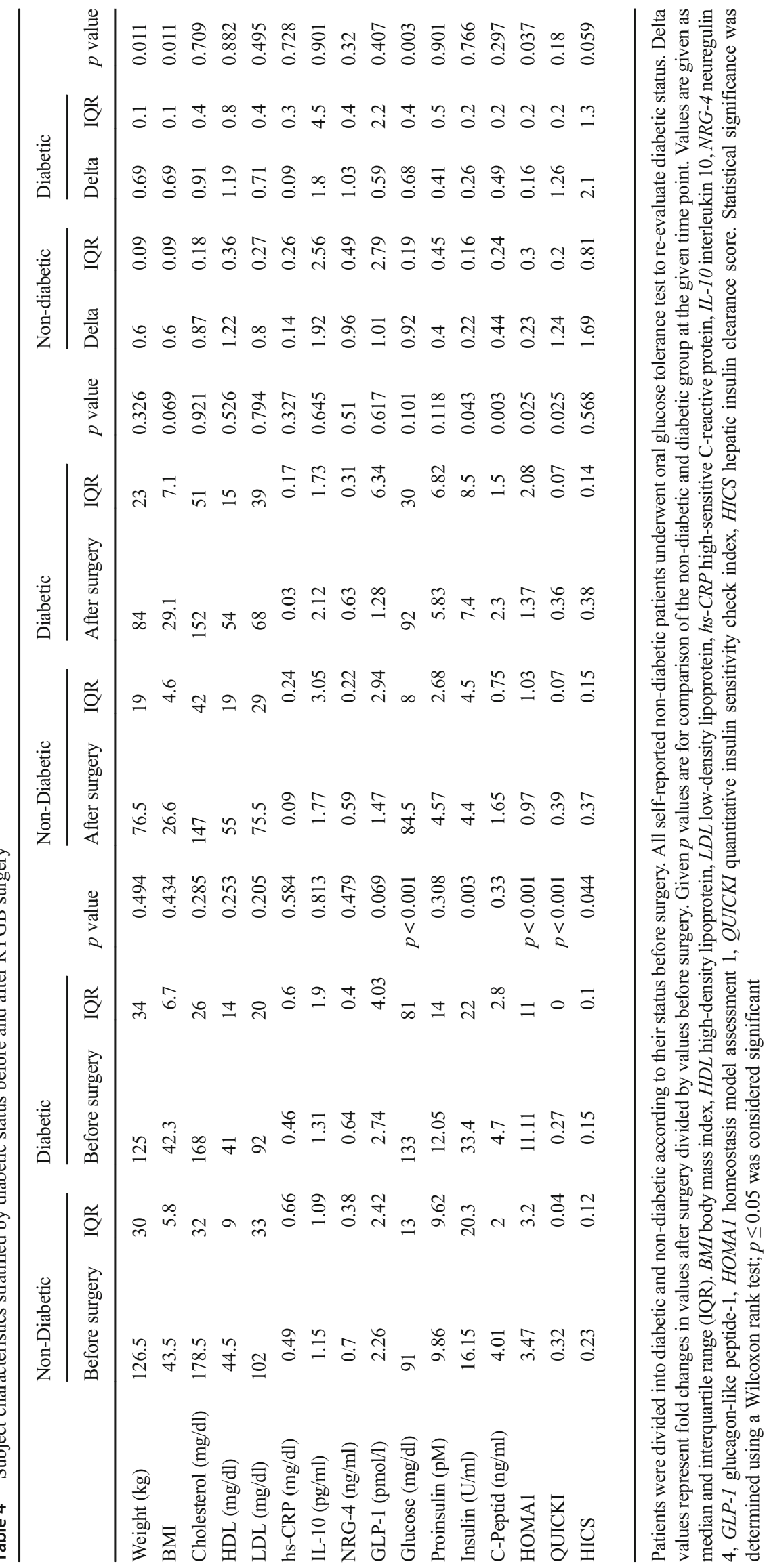


To further assess whether this observed amelioration of metabolic parameters would be reflected in reduced pancreatic dysfunction, we analyzed three miRNAs associated with the pancreas [30-32]. We observed a significant reduction in hsamiR-130b-3p $(p<0.001)$, and hsa-miR-132-3p $(p<0.001)$, (Fig. 1a, b). In contrast, hsa-miR-375 showed a significant increase 1 year after bariatric surgery $(p<0.001)$ (Fig. 1c). Furthermore, hsa-miR-375 baseline levels correlated with glucose levels $(R=0.482, p=0.01)$, insulin $(R=0.707$, $p<0.001)$, and C-peptide $(R=0.629, p<0.001)$ and consequently also with HOMA1 $(R=0.482, p=0.001)$. When separating patients according to their diabetic status, non-diabetic patients showed correlations only for insulin $(R=0.846$, $p<0.001)$ and C-peptide $(R=0.715, p<0.001)$ but not for glucose and HOMA1, whereas hsa-miR-375 was correlated to all of those parameters in diabetic patients (glucose $R=$ $0.514, p=0.035$; insulin $R=0.522, p=0.32$; C-peptide $R=$ $0.527, p=0.03$, and HOMA1 $R=0.693, p=0.002$ ). No correlations of hsa-miR-375 were observed 1 year after bariatric surgery regardless of patient status. Levels of all miRNAs were similar in patients regardless of their diabetic status before and after surgery. Likewise, reduction of miRNAs was similar regardless of diabetic status.

As our results indicated an increased hepatic insulin clearance after bariatric surgery and especially in diabetic patients, we analyzed markers of liver dysfunction (Table 5). Overall, liver dysfunction was reduced 1 year after bariatric surgery in all patients regardless of diabetic status. However, baseline $\gamma \mathrm{GT}$ levels were significantly higher in diabetic patients before surgery but similar after surgery (Table 6). $\gamma \mathrm{GT}$ levels correlated significantly with insulin levels in all patients $(R=$ $0.427, p=0.004)$. This correlation was only due to diabetic patients with a very strong correlation in these patients $(R=$ $0.743, p=0.001)$ and no correlation for non-diabetic patients $(R=-0.23, p=0.913)$. This correlation remained intact after bariatric surgery for all patients $(R=0.629, p<0.001)$ again with no correlation in non-diabetic patients $(R=0.027, p=$ $0.895)$ and a strong correlation for diabetic patients $(R=$ 0.929, $p<0.001)$.

Previously, hsa-miR-122-5p (miR-122) was associated with hepatic injury and insulin resistance [33]. We therefore determined if miR-122 was reduced after bariatric surgery. miR-122 was significantly downregulated 1 year after bariatric surgery in all patients (fold change $=0.45, p<0.001$ ) . Baseline levels and levels after bariatric surgery were similar in diabetic and non-diabetic patients. Hsa-miR122 correlated significantly at baseline with AST $(R=0.501, p=0.001)$, and $\operatorname{ALT}(R=0.555, p<0.001)$, but not with $\gamma \mathrm{GT}(R=0.158, p=$ $0.311)$. However, correlation of hsa-miR122 was significant for all liver parameters one year after bariatric surgery (AST: $R=0.44, p=0.003$; ALT: $R=0.303, p=0.48 ; \gamma \mathrm{GT}: R=$ $0.759, p<0.001)$. In addition, relative reduction of hsa-
Fig. 1 miRNA levels in morbidly obese patients before and after Roux-en-Y gastric bypass surgery. Circulating levels of miRNAs hsa-miR130 (panel a), hsa-miR-132 (panel b), hsa-miR375 (panel c), and hsa-miR122 (panel d) were determined as described under "Methods." RNA spike-in values were used for normalization of miRNA $C_{T}$ values to obtain delta- $C_{T}\left(\Delta C_{T}\right)$ values. Statistical significance was determined using a Wilcoxon rank test; $p \leq 0.05$ was considered significant
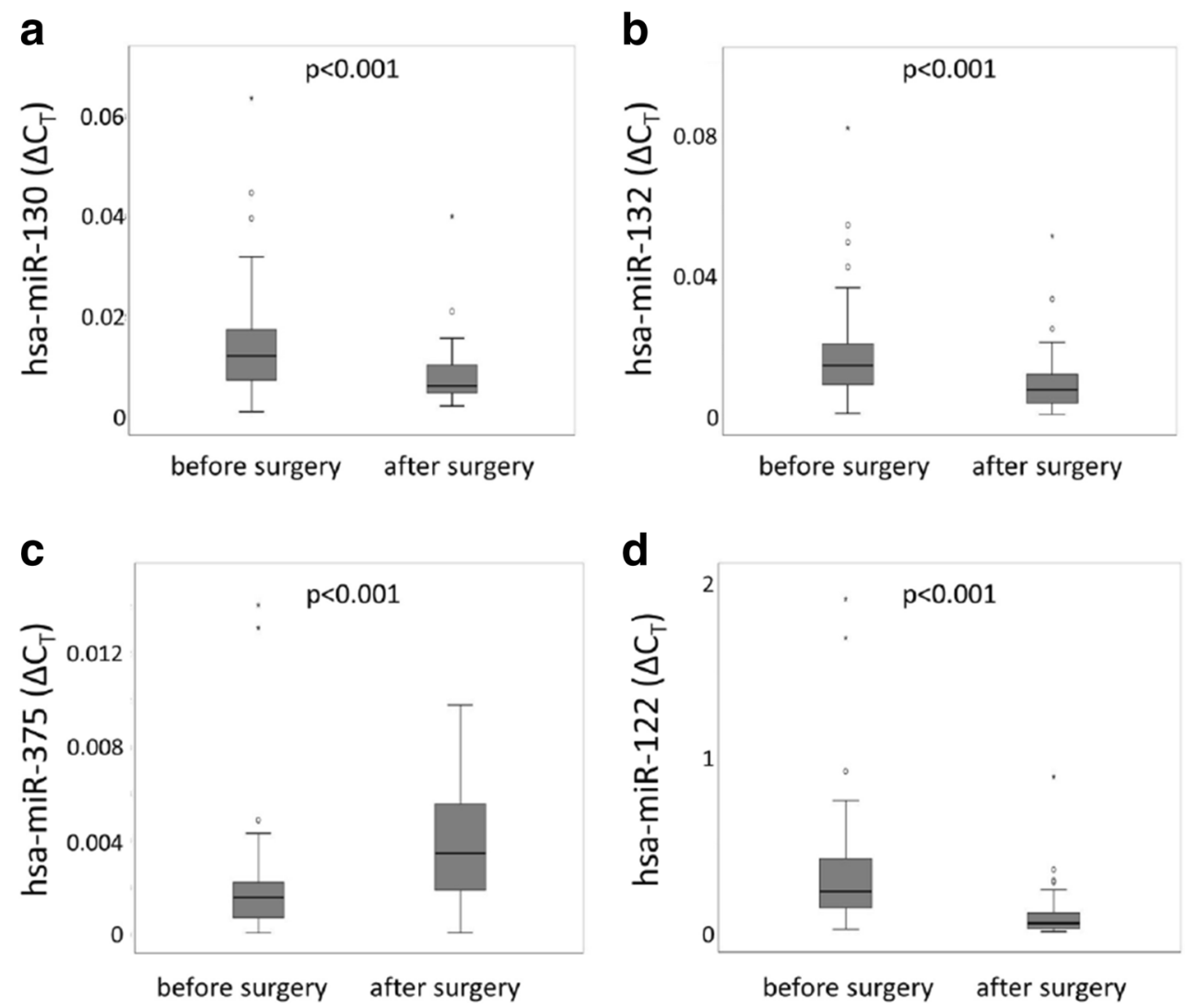

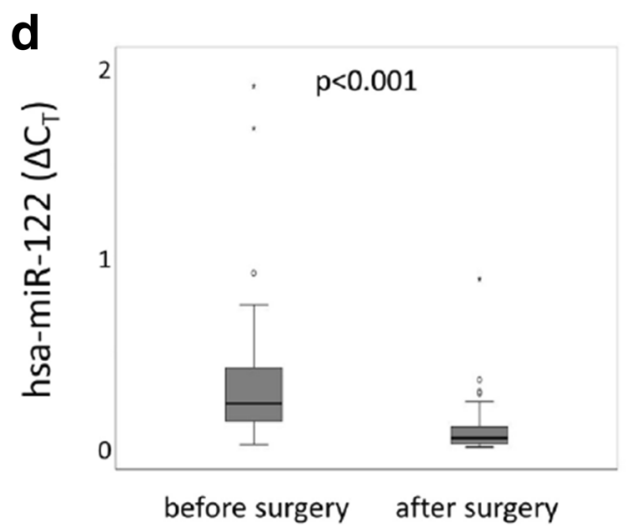


Table 5 Liver parameters in patients before and 1 year after gastric bypass surgery

\begin{tabular}{lllllllr}
\hline & Before surgery & IQR & After surgery & IQR & Delta & IQR & $p$ value \\
\hline AST (U/l) & 22 & 16 & 21 & 11 & 0.94 & 0.53 & 0.251 \\
ALT (U/1) & 29 & 37 & 19 & 12 & 0.65 & 0.89 & 0.002 \\
$\gamma$ GT (U/l) & 37 & 30 & 17 & 14 & 0.52 & 0.31 & $p<0.001$ \\
\hline
\end{tabular}

Hepatic liver enzyme parameters were measured in patients before and 1 year after bariatric surgery. Delta values represent fold changes in values after surgery divided by values before surgery. Values are given as median and interquartile range (IQR). AST aspartate transaminase, $A L T$ alanine transaminase, $\gamma G T \gamma$-glutamyltransferase. Statistical significance was determined using a Wilcoxon rank test; $p \leq 0.05$ was considered significant
miR122 correlated with relative reduction of AST $(R=0.378$, $p=0.012)$ and ALT $(R=0.303, p=0.48)$ but not with $\gamma \mathrm{GT}$ $(R=0.171, p=0.274)$. At baseline and 1 year after surgery, hsa-miR122 did not correlate with levels of metabolic syndrome. In addition, relative reduction of BMI 1 year after surgery did not correlate with miR-122 relative reduction levels. When analyzing only diabetic patients, we found no correlation of miR-122 levels before surgery but we observed a positive correlation of relative changes in QUICKI with relative changes in miR-122 $(R=0.535, p=0.027)$ in diabetic patients.

\section{Discussion}

We present here evidence for an amelioration of metabolic syndrome-associated parameters in 43 morbidly obese patients 1 year after bariatric surgery. The overall reduction can be observed in all patients regardless of previous diabetic status. In an additional patient group of 26 patients, we were able to confirm our results already 6 months after RYGB surgery. This suggests that morbidly obese patients regardless of their diabetic status benefit from RYGB surgery in terms of metabolic syndrome prevention or amelioration. Similar to previous studies, we observed a massive reduction in BMI
1 year after RYGB surgery [34]. In addition, lipid profiles changed with a reduction in total cholesterol and LDL and a slight increase in HDL. Furthermore, patients presented with a decreased proinflammatory status as suggested by the dramatic drop in hs-CRP and a similar increase in the anti-inflammatory cytokine IL10. The results obtained in our cohort are in line with previous experiences in RYGB surgery patients [35].

When analyzing markers of the metabolic syndrome, we found a robust and significant drop of glucose, insulin, C-peptide, and proinsulin levels regardless of diabetic status of the patients 1 year after surgery. As expected diabetic patients presented with higher levels of glucose, insulin, and C-peptide before surgery compared with nondiabetic patients. However, levels of proinsulin were not altered by the diabetic status. Previously, it was already suggested that high-fasting proinsulin levels might be indicative for insulin resistance [10]. Furthermore, high proinsulin secretion was previously associated with beta cell dysfunction [36]. The overall high levels of proinsulin might further suggest an overall dysfunction of beta cells in our patient cohort regardless of diabetic status before surgery. After RYGB surgery, similar levels of change for proinsulin and insulin levels as observed in our cohort were already reported [8]. We add to the current knowledge that changes in the level of proinsulin might be

Table 6 Liver parameters stratified by diabetic status before and 1 year after gastric bypass surgery

\begin{tabular}{|c|c|c|c|c|c|c|c|c|c|c|c|c|c|c|c|}
\hline & \multicolumn{2}{|c|}{ Non-Diabetic } & \multicolumn{2}{|l|}{ Diabetic } & \multirow[b]{2}{*}{$\begin{array}{l}p \\
\text { value }\end{array}$} & \multicolumn{2}{|c|}{ Non-Diabetic } & \multicolumn{2}{|l|}{ Diabetic } & \multirow[b]{2}{*}{$\begin{array}{l}p \\
\text { value }\end{array}$} & \multicolumn{2}{|c|}{$\begin{array}{l}\text { Non- } \\
\text { diabetic }\end{array}$} & \multicolumn{2}{|c|}{ Diabetic } & \multirow[b]{2}{*}{$\begin{array}{l}p \\
\text { value }\end{array}$} \\
\hline & $\begin{array}{l}\text { Before } \\
\text { surgery }\end{array}$ & IQR & $\begin{array}{l}\text { Before } \\
\text { surgery }\end{array}$ & IQR & & $\begin{array}{l}\text { After } \\
\text { surgery }\end{array}$ & IQR & $\begin{array}{l}\text { After } \\
\text { surgery }\end{array}$ & $\mathrm{IQR}$ & & Delta & IQR & Delta & $\mathrm{IQR}$ & \\
\hline $\begin{array}{l}\text { AST } \\
(\mathrm{U} / 1)\end{array}$ & 19.5 & 17 & 23 & 19 & 0.12 & 21 & 9 & 19 & 20 & 0.842 & 0.95 & 0.48 & 0.82 & 0.5 & 0.393 \\
\hline ALT (U/l) & 27.5 & 30 & 36 & 40 & 0.146 & 18.5 & 12.3 & 19 & 24.5 & 0.663 & 0.7 & 0.88 & 0.54 & 0.6 & 0.693 \\
\hline $\begin{array}{l}\gamma \mathrm{GT} \\
(\mathrm{U} / \mathrm{l})\end{array}$ & 28.5 & 28 & 45 & 33 & 0.04 & 15.5 & 12.5 & 17 & 14.5 & 0.357 & 0.53 & 0.32 & 0.5 & 0.3 & 0.799 \\
\hline
\end{tabular}

Patients were stratified according to their diabetic status and liver enzyme parameters are given accordingly. Delta values represent fold changes in values after surgery divided by values before surgery. Given $p$ values are for comparison of the non-diabetic and diabetic group at the given time point. Values are given as median and interquartile range (IQR). AST aspartate transaminase, $A L T$ alanine transaminase, $\gamma G T \gamma$-glutamyltransferase. Statistical significance was determined using a Wilcoxon rank test; $p \leq 0.05$ was considered significant 
directly connected to the overall weight loss after RYGB surgery in diabetic patients. In addition, we observed a dysfunctional relation of proinsulin with insulin and Cpeptide in morbidly obese patients before surgery as correlations of the three proteins were only present after RYGB surgery.

To further understand changes in the pancreatic function in morbidly obese individuals, we analyzed the profile of hsamiR-130b, hsa-miR-132-3p, and hsa-miR-375, representing three miRNAs associated with pancreatic function. hsa-miR$130 \mathrm{~b}$ was previously demonstrated to be elevated in islets from hyperglycemic human donors [30], hsa-miR-132-3p was proposed to be elevated long before the onset of diabetes in a mouse model, and hsa-miR-375 was demonstrated to be involved in the formation of beta cell identity, control of beta cell mass, and regulation of insulin secretion [32]. Our data indicates a reduction of hsa-miR-130b, and hsa-miR-132. Considering previous reports about these miRNAs, such reduction might indeed indicate reduced pancreatic stress after RYGB surgery due to the reduction of hyperglycemia. We speculate that both hsa-miR-130 and hsa-miR-132 might be early indicators of metabolic stress before the onset of clinical symptoms as also the non-diabetic patient group displayed a significant reduction in both miRNAs 1 year after surgery. Furthermore, we observed an increase of hsa-miR-375 1 year after bariatric surgery. Knockout of hsa-miR-375 in a mouse model induced a hyperglycemic phenotype with reduced beta cell mass [37]. The increased level of circulating hsa-miR-375 might therefore indicate an amelioration of beta cell function or changes in beta cell mass as indicated previously [14].

Previous research suggested NRG-4 as a potential marker of metabolic syndrome [38] including elevation in prediabetic and diabetic patients [39]. However, within our cohort, we did not detect differences in plasma levels between non-diabetic and diabetic patients and failed to measure changes 1 year after bariatric surgery. However, recent data from mouse experiments described a potential role of hepatic NRG-4 in gluconeogenesis [29]. As we measured only plasma levels of NRG-4, we cannot rule out different organs being responsible for its production before and after RYGB surgery.

GLP-1 was reported to be increasingly released postprandial after RYGB and contribute to improved glucose metabolism [40]. At baseline in a fasting state, we were not able to determine changes in circulating levels of GLP-1. However, GLP-1 secretion was reported to be dependent on the rate of carbohydrate digestion [41] which could explain the lack of GLP-1 change in our cohort as blood samples were taken in an overnight fasting state.

RYGB surgery was previously associated with an increase in hepatic insulin clearance [28]. Our study supports this data as we were able to observe a similar increase of hepatic insulin clearance measured via the ratio of C-peptide and insulin. This increased hepatic insulin clearance might also explain the beneficial development of the HOMA index [42]. We therefore suggest that especially diabetic patients benefit from bariatric surgery by increasing insulin clearance. Besides a possible amelioration of hepatic insulin clearance, overall liver dysfunction was reduced after RYGB surgery. Both previously elevated liver enzyme parameters were reduced in our cohort 1 year after surgery. Of note, diabetic patients presented with elevated $\gamma$ GT levels compared with non-diabetic patients in our cohort. Furthermore, $\gamma$ GT levels correlated significantly with insulin levels before and after bariatric surgery exclusively in diabetic patients. $\gamma$ GT was already shown to associate with type 2 diabetes mellitus and metabolic syndrome [43]. Furthermore, $\gamma$ GT was already demonstrated to correlate with several markers of obesity and metabolic syndrome [44, 45]. Our data might indicate that diabetic status and liver function might be closely related in diabetic patients even after RYGB surgery.

To further understand changes in liver function after surgery, we analyzed hsa-miR-122. Altered hsa-miR-122 function has been already documented in a variety of liver diseases including alcohol-induced liver disease and nonalcoholic fatty liver disease as well as in insulin resistance [33, 46, 47]. Recently, this miRNA was associated with the prediction of liver dysfunction after liver resection [48]. Furthermore, previous publications suggested a role for hsa-miR-122 as a biomarker for obesity [49]. Here we were able to demonstrate that patients showed reduced levels of hsa-miR122 1 year after RYGB surgery regardless of diabetic status. Interestingly, hsa-miR-122 correlated with AST and ALT but not $\gamma$ GT before surgery.

Some limitations might be considered when interpreting data from our cohort group. We only included patients undergoing RYGB surgery omitting other bariatric surgery procedures. Given the success of the procedure for weight loss, a stratification for efficiency is difficult as all patients massively reduced their BMI 1 year after the surgery. It would be interesting to evaluate in long-term studies the overall health benefit and changes in the metabolic risk in patients undergoing RYGB surgery. In addition, due to legal restrictions, we could not access data of patients who declined to participate in the study. Therefore, a selection bias between those patients who volunteered to participate in the study and those who declined to take part cannot be excluded. Similarly, a selection bias might exist between those patients who voluntarily performed the required follow-up visits and those who did not.

However, overall, our data demonstrates that all morbidly obese individuals benefited from RYGB surgery regardless of previous diabetic status. Classical parameters of the metabolic syndrome, but also miRNAs associated with hepatic and pancreatic stress, were all reduced in our cohort. We therefore suggest, that, at least in our cohort, no metabolically healthy individual was present as all patients showed improvement in metabolic syndrome-associated parameters. 
Funding Information Open access funding provided by Medical University of Vienna. This work was financed by a grant from the Austrian National Bank (Jubilaeumsfondsprojekt No. 16377) to GRK.

\section{Compliance with Ethical Standards}

Conflict of Interest The authors declare that they have no conflict of interest.

Ethical Approval The study was approved by the Ethical Committee of the participating hospital.

Informed Consent Written informed consent was obtained for each participant before bariatric surgery in the study.

Open Access This article is distributed under the terms of the Creative Commons Attribution 4.0 International License (http:// creativecommons.org/licenses/by/4.0/), which permits unrestricted use, distribution, and reproduction in any medium, provided you give appropriate credit to the original author(s) and the source, provide a link to the Creative Commons license, and indicate if changes were made.

\section{References}

1. Pivovarova O, Bernigau W, Bobbert T, et al. Hepatic insulin clearance is closely related to metabolic syndrome components. Diabetes Care. 2013;36(11):3779-85.

2. Reaven GM. Banting lecture 1988. Role of insulin resistance in human disease. Diabetes. 1988;37(12):1595-607.

3. Mokdad AH, Bowman BA, Ford ES, et al. The continuing epidemics of obesity and diabetes in the United States. Jama. 2001;286(10):1195-200.

4. Kahn SE, Prigeon RL, McCulloch DK, et al. Quantification of the relationship between insulin sensitivity and beta-cell function in human subjects. Evidence for a hyperbolic function. Diabetes. 1993;42(11):1663-72

5. Le Stunff C, Bougneres P. Early changes in postprandial insulin secretion, not in insulin sensitivity, characterize juvenile obesity. Diabetes. 1994;43(5):696-702.

6. Bojsen-Moller KN, Lundsgaard AM, Madsbad S, et al. Hepatic insulin clearance in regulation of systemic insulin concentrationsrole of carbohydrate and energy availability. Diabetes. 2018;67(11): 2129-36.

7. Gumbs AA, Modlin IM, Ballantyne GH. Changes in insulin resistance following bariatric surgery: role of caloric restriction and weight loss. Obes Surg. 2005;15(4):462-73.

8. Johansson HE, Haenni A, Ohrvall M, et al. Alterations in proinsulin and insulin dynamics, HDL cholesterol and ALT after gastric bypass surgery. A 42-months follow-up study. Obes Surg. 2009;19(5): 601-7.

9. Wickremesekera K, Miller G, Naotunne TD, et al. Loss of insulin resistance after Roux-en-Y gastric bypass surgery: a time course study. Obes Surg. 2005;15(4):474-81.

10. Johansson HE, Ohrvall M, Haenni A, et al. Gastric bypass alters the dynamics and metabolic effects of insulin and proinsulin secretion. Diabet Med: a journal of the British Diabetic Association. 2007;24(11):1213-20.

11. Hohensinner PJ, Kaun C, Ebenbauer B, et al. Reduction of premature aging markers after gastric bypass surgery in morbidly obese patients. Obes Surg. 2018;28(9):2804-10.
12. Rega-Kaun G, Kaun C, Ebenbauer B, et al. Bariatric surgery in morbidly obese individuals affects plasma levels of protein $\mathrm{C}$ and thrombomodulin. J Thromb Thrombolysis. 2019;47(1):51-6.

13. Matsuda A, Makino N, Tozawa T, et al. Pancreatic fat accumulation, fibrosis, and acinar cell injury in the Zucker diabetic fatty rat fed a chronic high-fat diet. Pancreas. 2014;43(5):735-43.

14. Lautenbach A, Wernecke M, Riedel N, et al. Adaptive changes in pancreas post Roux-en-Y gastric bypass induced weight loss. Diabetes Metab Res Rev. 2018;34(7):e3025.

15. Sullivan CA, Cacicedo JM, Rajendran I, et al. Comparison of proinsulin and C-peptide secretion in healthy versus long-standing type 1 diabetes mellitus cohorts: a pilot study. PLoS One. 2018;13(11): $\mathrm{e} 0207065$.

16. Uchizono Y, Alarcon C, Wicksteed BL, et al. The balance between proinsulin biosynthesis and insulin secretion: where can imbalance lead? Diabet Obes Metab. 2007;9(Suppl 2):56-66.

17. Rhodes CJ. Type 2 diabetes-a matter of beta-cell life and death? Science. 2005;307(5708):380-4.

18. Kahn SE, Halban PA. Release of incompletely processed proinsulin is the cause of the disproportionate proinsulinemia of NIDDM. Diabetes. 1997;46(11):1725-32.

19. Watkins RA, Evans-Molina C, Terrell JK, et al. Proinsulin and heat shock protein 90 as biomarkers of beta-cell stress in the early period after onset of type 1 diabetes. Transl Res. 2016;168:96-1060.

20. Kruschitz R, Luger M, Kienbacher C, et al. The effect of roux-en-Y vs. omega-loop gastric bypass on liver, metabolic parameters, and weight loss. Obes Surg. 2016;26(9):2204-12.

21. Llave C, Xie Z, Kasschau KD, et al. Cleavage of Scarecrow-like mRNA targets directed by a class of Arabidopsis miRNA. Science. 2002;297(5589):2053-6.

22. Victoria B, Nunez Lopez YO, Masternak MM. MicroRNAs and the metabolic hallmarks of aging. Mol Cell Endocrinol. 2017;455:13147.

23. Runkel N, Colombo-Benkmann M, Hüttl TP, et al. Evidence-based German guidelines for surgery for obesity. Int J Color Dis. 2011;26(4):397-404.

24. Disse E, Pasquer A, Espalieu P, et al. Greater weight loss with the omega loop bypass compared to the Roux-en-Y gastric bypass: a comparative study. Obes Surg. 2014;24(6):841-6.

25. Blondal T, Jensby Nielsen S, Baker A, et al. Assessing sample and miRNA profile quality in serum and plasma or other biofluids. Methods. 2013;59(1):S1-6.

26. Matthews DR, Hosker JP, Rudenski AS, et al. Homeostasis model assessment: insulin resistance and beta-cell function from fasting plasma glucose and insulin concentrations in man. Diabetologia. 1985;28(7):412-9.

27. Katz A, Nambi SS, Mather K, et al. Quantitative insulin sensitivity check index: a simple, accurate method for assessing insulin sensitivity in humans. J Clin Endocrinol Metab. 2000;85(7):2402-10.

28. Bojsen-Moller KN, Dirksen C, Jorgensen NB, et al. Increased hepatic insulin clearance after Roux-en-Y gastric bypass. J Clin Endocrinol Metab. 2013;98(6):E1066-71.

29. Zhang L, Bai M, Tang H, et al. Role of hepatic neuregulin 4 in the regulation of gluconeogenesis in mice. Life Sci. 2019;217:185-92.

30. Ofori JK, Salunkhe VA, Bagge A, et al. Elevated miR-130a/ miR130b/miR-152 expression reduces intracellular ATP levels in the pancreatic beta cell. Sci Rep. 2017;7:44986.

31. Seyhan AA, Nunez Lopez YO, Xie H, et al. Pancreas-enriched miRNAs are altered in the circulation of subjects with diabetes: a pilot cross-sectional study. Sci Rep. 2016;6:31479.

32. Eliasson L. The small RNA miR-375 - a pancreatic islet abundant miRNA with multiple roles in endocrine beta cell function. Mol Cell Endocrinol. 2017;456:95-101.

33. Iacomino G, Siani A. Role of microRNAs in obesity and obesityrelated diseases. Genes Nutr. 2017;12:23. 
34. Courcoulas AP, Christian NJ, Belle SH, et al. Weight change and health outcomes at 3 years after bariatric surgery among individuals with severe obesity. Jama. 2013;310(22):2416-25.

35. Barazzoni R, Palmisano S, Cappellari G, et al. Gastric bypassinduced weight loss alters obesity-associated patterns of plasma pentraxin-3 and systemic inflammatory markers. Surg Obes Relat Dis. 2016;12(1):23-32.

36. Sims EK, Bahnson HT, Nyalwidhe J, et al. Proinsulin secretion is a persistent feature of type 1 diabetes. Diabetes Care. 2019;42(2): 258-64.

37. Poy MN, Hausser J, Trajkovski M, et al. miR-375 maintains normal pancreatic alpha- and beta-cell mass. Proc Natl Acad Sci U S A. 2009;106(14):5813-8.

38. Cai $\mathrm{C}, \mathrm{Lin} \mathrm{M}, \mathrm{Xu} \mathrm{Y}$, et al. Association of circulating neuregulin 4 with metabolic syndrome in obese adults: a cross-sectional study. BMC Med. 2016;14(1):165.

39. Chen LL, Peng MM, Zhang JY, et al. Elevated circulating Neuregulin4 level in patients with diabetes. Diabetes Metab Res Rev. 2017;33:4.

40. Jorgensen NB, Dirksen C, Bojsen-Moller KN, et al. Exaggerated glucagon-like peptide 1 response is important for improved betacell function and glucose tolerance after Roux-en-Y gastric bypass in patients with type 2 diabetes. Diabetes. 2013;62(9):3044-52.

41. Martinussen C, Bojsen-Moller KN, Dirksen C, et al. Augmented GLP-1 secretion as seen after gastric bypass may be obtained by delaying carbohydrate digestion. J Clin Endocrinol Metab. 2019;104(8):3233-44.
42. Dirksen C, Jorgensen NB, Bojsen-Moller KN, et al. Mechanisms of improved glycaemic control after Roux-en-Y gastric bypass. Diabetologia. 2012;55(7):1890-901.

43. Kunutsor SK. Gamma-glutamyltransferase-friend or foe within? Liver Int: official journal of the International Association for the Study of the Liver. 2016;36(12):1723-34.

44. Nilssen O, Forde OH. Seven-year longitudinal population study of change in gamma-glutamyltransferase: the Tromso Study. Am J Epidemiol. 1994;139(8):787-92.

45. Nilssen O, Forde OH, Brenn T. The Tromso Study. Distribution and population determinants of gamma-glutamyltransferase. Am J Epidemiol. 1990;132(2):318-26.

46. Satishchandran A, Ambade A, Rao S, et al. MicroRNA 122, regulated by GRLH2, protects livers of mice and patients from ethanolinduced liver disease. Gastroenterology. 2018;154(1):238-52 e7.

47. Zhang Y, Jia Y, Zheng R, et al. Plasma microRNA-122 as a biomarker for viral-, alcohol-, and chemical-related hepatic diseases. Clin Chem. 2010;56(12):1830-8.

48. Starlinger P, Hackl H, Pereyra D, et al. Predicting postoperative liver dysfunction based on blood derived microRNA signatures. Hepatology. 2019;69(6):2636-51.

49. Jones A, Danielson KM, Benton MC, et al. miRNA signatures of insulin resistance in obesity. Obesity. 2017;25(10):1734-44.

Publisher's Note Springer Nature remains neutral with regard to jurisdictional claims in published maps and institutional affiliations. 